\title{
“COMPUTABILIDADE DE UM MÉTODO DE EXTRAÇÃO DO GRAU DE EVIDÊNCIA A PARTIR DAS FREQUÊNCIAS E DESVIOS"
}

Eduardo Mário Dias ${ }^{1}$, Paulo Roberto Schroeder de Souza ${ }^{2}$, Paulo de Mattos Pimenta ${ }^{3}$, Maria Lidia Rebello Pinho Dias ${ }^{4}$, Caio Fernando Fontana ${ }^{5}$

${ }^{1}$ PEA - Grupo de Automação Elétrica em Sistemas Industriais-GAESI, Engenharia Elétrica da Escola Politécnica da Universidade de São Paulo (emdias@ pea.usp.br)

${ }^{2}$ Departamento de Engenharia de Estruturas e Fundações - Laboratório Mecânica Computacional, Engenharia Civil da Escola Politécnica da Universidade de São Paulo (f.rlara.dir@centropaulasouza.sp.gov.br)

${ }^{3}$ Departamento de Engenharia de Estruturas e Fundações - Laboratório Mecânica Computacional, Engenharia Civil da Escola Politécnica da Universidade de São Paulo (ppimenta@usp.sp.gov.br)

${ }^{4}$ PEA - Grupo de Automação Elétrica em Sistemas Industriais-GAESI, Engenharia Elétrica da Escola Politécnica da Universidade de São Paulo (lidia.rebello.dias@ gmail.com)

${ }^{5}$ PEA - Grupo de Automação Elétrica em Sistemas Industriais-GAESI, Engenharia Elétrica da Escola Politécnica da Universidade de São Paulo (caio_fontana@pea.usp.br)

Resumo. Nesse trabalho, a partir dos valores estatísticos de frequência serão extraídos os dados que, após receberem um tratamento lógico paraconsistente, resultarão em Graus de Evidência. Para a demonstração desse método utilizaremos o gráfico que mostra as variações de frequência e Valores em estudo específicos medidos em um determinado experimento, contendo banco de dados definido nestes estudos. O método de determinação de Grau Evidência utilizando valores estatísticos se refere ao cálculo de variáveis de distribuição Normal com aplicações da Lógica Paraconsistente Anotada (LPA). Essa metodologia se destina a determinar o Grau de Evidência Favorável do estudo especifico. Para a extração do Grau de Evidência, iremos a partir do resultado gráfico obtido, fazer a caracterização das curvas com simetria em torno da Média. Para abranger um intervalo que compreende praticamente $100 \%$ de probabilidade sob a curva vamos considerar como análise além da Média, o triplo do Desvio Padrão. O sistema deve ser capaz de apresentar boa computabilidade dos dados envolvidos. No tratamento de incertezas, os valores devem ser computacionalmente factíveis para o sistema criar regras de inferências e obter conclusões. No tratamento de dados com valores incertos, o sistema deve viabilizar a combinação de avaliações qualitativas com valores quantitativos de incerteza. 
Palavras-Chave: paraconsistente; evidência; frequência; desvio padrão; computabilidade.

\begin{abstract}
In this work, from the statistical values of frequency are extracted data that, after receiving a paraconsistent logical treatment, will result in degrees of evidence. For the demonstration of this method we will use the chart that shows the changes in frequency and specific study measured Values in a given experiment containing database defined in these studies. The method of determination of Grade Evidence using statistical values concerning the calculation of Normal distribution variables with applications of Paraconsistent Logic Annotated (LPA). This methodology is intended to determine the degree of Evidence in favour of specific study. For the extraction of degree of Evidence, we will from the graphic result obtained, making the characterization of curves with symmetry around the average. To span a range that includes almost $100 \%$ probability under the curve Let's consider as analysis beyond the average, three times the standard deviation. In the treatment of uncertainties, the values must be computationally feasible to create rules of inferences and derive conclusions. In the processing of data with uncertain values, the system should enable the combination of qualitative with quantitative values of uncertainty.
\end{abstract}

Keywords: paraconsistent; evidence; frequency; standard deviation; Computability.

\title{
1. Introdução
}

No início de uma investigação, e em Medicina não se foge à regra, frente aos dados disponíveis é necessário se fazer uma escolha racional sobre o método particular de análise que será utilizado. No processo da escolha são feitas algumas considerações importantes em relação ao objetivo da investigação, as características matemáticas das variáveis envolvidas; as hipóteses estatísticas feitas sobre estas variáveis; bem como a melhor forma de recolhimento dos dados. Além disso, segundo Marques e Dutra [12] dentro do contexto dos sistemas que agem racionalmente, duas abordagens principais podem ser utilizadas: raciocínio lógico e raciocínio probabilístico. O raciocínio lógico pondera sobre o conhecimento prévio a respeito do problema e sobre essa base de conhecimento retira suas conclusões. Essa abordagem, apesar de poderosa, pode não ser útil em situações em que não se conhece previamente todo o escopo do problema. Para estes casos, o raciocínio probabilístico surge como uma boa opção.

Lógica Paraconsistente Anotada (LPA) é uma Lógica não clássica. LPA é uma classe de Lógica Paraconsistente que possui um reticulado associado que desempenha um papel sumamente importante na sua representação. [5]

A LPA é uma classe de lógica Paraconsistente Evidencial que faz tratamento de sinais associados à anotações que permitem descrição e equacionamento por meio de Algoritmos.[1] 
$\mathrm{Na}$ LPA as fórmulas proposicionais vêm acompanhadas de anotações. Cada anotação $\mu$, pertencente a um reticulado finito ${ }^{t}$ atribui valores à sua correspondente fórmula proposicional $\rho$. Considerando uma lógica evidencial, as anotações vêm representadas por Graus de Evidência ou Crença. Quando as proposições vêm acompanhadas de Anotações, ou Graus de Evidência, é possível a aplicação Real da Lógica Paraconsistente em Sistemas de Análise e tomada de decisão. A possibilidade de aplicar a Lógica Paraconsistente Anotada em áreas de Inteligência Artificial e correlatas transforma-a em forte ferramenta para o tratamento do conhecimento incerto, incompleto ou inconsistente em I. A. [10]

O raciocínio probabilístico trata o grau de incerteza associado à maioria dos domínios, e tem as Redes Bayesianas como uma boa ferramenta usada em muitas aplicações do mundo real para este tipo de raciocínio. Na área de medicina, o raciocínio probabilístico e as Redes Bayesianas são bastante utilizados em sistemas de apoio à decisão, como por exemplo em testes epidemiológicos que são a base da investigação para o diagnóstico precoce de algumas doenças. Existem testes baseados em observações de determinado fenômeno, ou através de técnicas laboratoriais, que permitem a previsão ou detecção desse acontecimento numa fase incipiente de desenvolvimento.[16]

O objetivo principal é a apresentação de uma nova abordagem para os Métodos Quantitativos em Medicina para Sistemas de Apoio à Decisão, em que é feita a junção da teoria probabilística, usada tradicionalmente através da teoria de Bayes e a Lógica Paraconsistente Anotada.

Neste artigo será apresentada a técnica para apoio a decisão médica que envolvem aplicações diretas dos fundamentos da Lógica Paraconsistente Anotada com dois valores (LPA2v), uma das metodologias mais interativas e em desenvolvimento da Inteligência Artificial (I.A.), mesclado aos estudos estatísticos aplicando processos de mecânica computacional.[1]

Para esse estudo será utilizado um dos vários modos de aplicação da LPA2v para extração do Grau de Evidência de um banco de dados, como "Extração do Grau de Evidência a partir das Frequência e Desvios".

Para este modo de aplicação, a partir dos valores estatísticos de gráficos de frequência da glicemia populacional, serão extraídos os dados que, após receberem um tratamento lógico paraconsistente, resultarão em Graus de Evidência $\mu_{\mathrm{EF}}$. [4]

Nesse trabalho, a técnica utilizada para extração de Graus de Evidência resultará em valores que servirão de apoio ao diagnóstico médico referente aos pacientes com risco de serem portadores de moléstias. Ao final será mostrada uma pequena Rede de Análise 
Paraconsistente capaz de analisar os resultados obtidos, promovendo um sinal resultante evidencial, para aumentar o Grau de Confiabilidade da decisão.

\subsection{BANCO DE DADOS ÍNDIAS PIMA}

Para a aplicação das técnicas LPA2v, foi escolhido o banco de dados para estudos práticos de Apoio a Decisão Médica (ADM) referente a uma população de mulheres Índias Pima (Pima Indians ${ }^{l}$ ) com prevalência elevada em Diabetes Mellitus Gestacional, residentes próximo a Phoenix, Arizona, USA. A razão da escolha é que Pima Indians trata-se de um exemplo clássico, utilizado como padrão em diversos trabalhos e refere-se a estudo feito segundo critérios da Organização Mundial da Saúde (World Health Organization - WHO).

Todo o processo de extração de Graus de Evidência será feito por meio de Análise das informações do Prontuário Eletrônico dos Pacientes (PEP) da Pima Indians. [14]

As informações utilizadas são aquelas aonde estão expostas as possíveis causas que levaram a resultados para apoio a decisão médica por meios tradicionais. Nas informações expostas nos prontuários, com base no trabalho mostrado em [13], foram classificados as pacientes de sexo feminino como diabética ou não-diabética segundo 8 critérios, os quais são considerados como as entradas listadas para o Banco de dados.

\subsection{LEVANTAMENTO ESTATÍSTICO}

A base de dados Índias Pima é formada por 768 registros (indivíduos), com 8 atributos com informações médicas, mais um que indica o diagnóstico (classe ou atributo objetivo):

(1) NUMPREGNAN: números de vezes que ficou grávida

(2) PLASMAGLUC: concentração de glicose no plasma em teste de tolerância de glicose oral de 2 horas (Considerações para jejum, $1 \mathrm{~h}$ e $2 \mathrm{~h}$ )

(3) DIASTOLICB pressão sanguínea mínima (diastólica) $(\mathrm{mm} / \mathrm{Hg})$

(4) TRICEPSSKI: dobras na pele do tríceps (mm)

(5) TWOHRSERUM: aplicações de 2 horas de soro com insulina (um U/ml)

(6) BODYMASSIN: índice massa corpórea (peso em $\mathrm{Kg} /($ altura em m²)

(7) DIABETESPE: função de genealogia de diabetes

(8) AGE: idade (anos)

\footnotetext{
$1 \quad$ Pima Indians Diabetes Database, fornecida pela John Hopkins University, 1990, e bastante utilizada na literatura para comparação entre metodologias e como base para trabalhos diversos.
} 
$\mathrm{Na}$ base de dados foram registradas pacientes observadas que apresentavam sinais de diabetes ou não. A análise feita no item (2) [14], foi realizada seguindo o critério do Teste Oral de Tolerância a Glicose (TOTG), 75 mg/dl no plasma, em seguida são tomadas as medidas de Glicemia em jejum, após 1 hora, e após 2 horas. Esta é uma aplicação de plasma glicosilada para exames de rotina em gestantes entre 24 e 28 semanas de gravidez. Atualmente, por prevenção, tem-se feito esses testes a partir da 20. ${ }^{a}$ semana de gestação.

A TOTG é um dos testes mais sugeridos para tomada de decisão médica pela International Committee (IEC), National Diabetes Data Group e subsequente aprovação da Organização Mundial da Saúde (OMS). Esse teste parte do princípio da Glicemia em Jejum Alterada (GJA) e Intolerância a Glicose (IG). [9]

Características consideradas dos parâmetros dos itens:

a) ITEM 1: NUMPREGNAN

Quantidade de mulheres grávidas $\leq 1$

Quanto menor o número de vezes que ficou grávida maior é o fator de risco.

b) ITEM 2:

$110 \mathrm{mg} / \mathrm{dl} \leq$ Normal em jejum

Teste jejum $\geq 110=$ diabética

Teste após $1 \mathrm{~h} \geq 140=$ diabética

Teste após $2 \mathrm{~h} \geq 200=$ diabética

c) ITEM 3:

Hipertensão

Pressão sanguínea Diastólica $(\mathrm{mm} \mathrm{Hg}) \geq 90$

Pressão Sistólica $(\mathrm{mm} \mathrm{Hg}) \geq 140$

(fatores de Risco)

d) ITEM 4:

Dobras na pele do tríceps ( $\mathrm{mm}$ )

Valores críticos para $\geq 4,5 \mathrm{~mm}$

e) ITEM 5:

Valores para exame de resistência a insulina podemos considerar $\geq 95 \mathrm{mg} / \mathrm{dl}$

f) ITEM 6: ÍNDICE DE MASSA CORPÓREA - IMC

(peso em kg/(altura em $\left.\mathrm{cm}^{2}\right)$ )

Sobrepeso $=(25-29,9)$

Obeso $=(30-39,9)$

Obeso Mórbido $\geq 40 \mathrm{Kg} / \mathrm{cm}^{2}$ 


\section{g) ITEM 7:}

Função de genealogia de diabetes na família.

Quantidade de parentes diabéticos

O fator crítico

\section{h) ITEM 8:}

Mulheres maiores que 21 anos de idade temos um fator de risco crescente por idade

(*) massa de dados, Banco de Dados (BD): diabetes.dbf . [14]

\subsection{RESULTADOS DO PROCESSO ESTATÍSTICO}

A partir dos valores do banco de dados (Índias Pima), foram levantadas as curvas Normais da Frequência x Glicemia como um dos modelos de gráficos que consta neste segmento, que nos mostram todas as variações dos Pontos de Corte escolhidos pelo autor, referentes à população de Índias PIMA sadia ou doente.

Nesse processo estatístico foi utilizado o Software MINITAB Versão 15 (MINITAB, 2008).

Na construção de cada gráfico, foi utilizado o processo de Tabulação Cruzada.

Para a obtenção das tabelas $2 \times 2$ do tipo Figura 1 [14] seguiu-se o mesmo processo e com os mesmos pontos de Corte escolhidos anteriormente.

Um dos HISTOGRAMAS DE FREQUENCIA X GLICEMIA, figura 1, (dados utilizados: indias PIMA faixa de ponto de corte: 90 - $200 \mathrm{mg} / \mathrm{dl}$ - FONTE: (MINITAB Versão 15, 2008) normal aplicando FREQUENCIA X GLICEMIA)

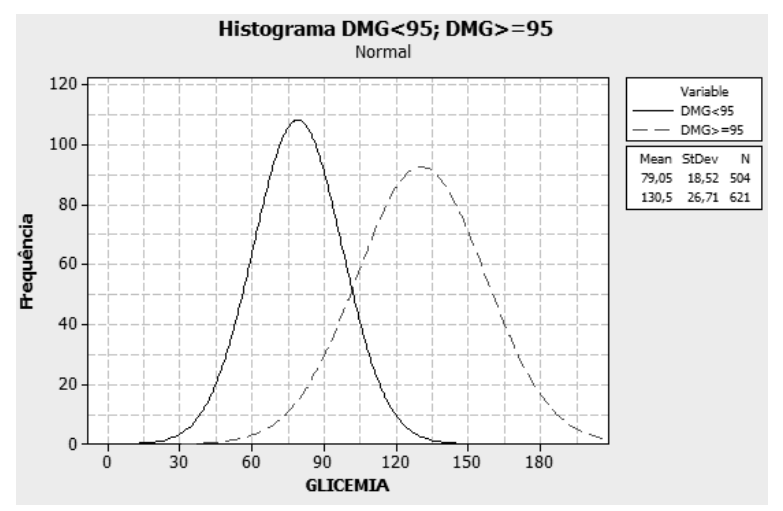

Figura 1. HISTOGRAMAS DE FREQUENCIA X GLICEMIA

Após a construção das tabelas para cada uma, ficaram disponibilizados os seguintes valores: verdadeiro positivo, falso negativo, verdadeiro negativo, falso positivo. 
Para efetuar o levantamento estatístico foi utilizado um software específico, desenvolvido pelo Autor em Linguagem de programação C (APÊNDICE A). Com esse programa foram calculados os valores da: Prevalência, Sensibilidade, Especificidade, Falso Positivo (1 - Especificidade) para cada tabela.

A Prevalência considerada foi a calculada para cada tabela, como exemplifica parcialmente a Tabela 1 (SOUZA, 2009) em relação ao seu Ponto de Corte (PC), que fornece um valor de análise coerente para que possam ser utilizados os resultados para Apoio a Decisão Médica (ADM).

Tabela 1. Tabulação cruzada (demonstração parcial) com valores calculados de sensibilidade, especificidade, prevalência e falso-positivo.

Tabelas $2 \times 2$ geradas pelo minitab entre pontos de corte:

$90>=\mathrm{pc}<=200$, valor de prevalência para $\mathrm{pc}=100 \Rightarrow \mathrm{p}=0.743490 \Rightarrow 74,3490 \%$. [14]

\begin{tabular}{|ccccccccccc}
\hline N & & $\mathbf{0}$ & 1 & PC & Sensibilidade & Especificidade & Prevalência & $\begin{array}{c}\text { Falso- } \\
\text { positivo }\end{array}$ & $\mu$ ER \\
& & & & & & & & & & \\
1 & 0 & 104 & 93 & 90 & 1.000000 & 0.527919 & 0.743490 & 0.472081 & 0.721 \\
& 1 & 0 & 571 & & & & & & \\
2 & 0 & 147 & 50 & 95 & 1.000000 & 0.746193 & 0.773041 & 0.253807 & 0.616 \\
& 1 & 0 & 671 & & & & & & \\
3 & 0 & 197 & 0 & 100 & 1.000000 & 1.000000 & 0.743490 & 0.000000 & 0.492 \\
& 1 & 0 & 571 & & & & & & \\
& & & & & & & & & \\
& & & & & & & & & \\
23 & 0 & 197 & 0 & 200 & 1.000000 & 1.00000 & 0.743490 & 0.000000 & 0.492 \\
& 1 & 0 & 571 & & & & & & \\
23 & 0 & 197 & 0 & 200 & 1.000000 & 1.00000 & 0.743490 & 0.000000 & 0.492 \\
& 1 & 0 & 571 & & & & & & \\
23 & 0 & 197 & 0 & 200 & 1.000000 & 1.00000 & 0.743490 & 0.000000 & 0.492 \\
& 1 & 0 & 571 & & & & & & \\
23 & 0 & 197 & 0 & 200 & 1.000000 & 1.00000 & 0.743490 & 0.000000 & 0.492 \\
& 1 & 0 & 571 & & & & & & \\
\hline
\end{tabular}

Para obtenção de uma ampla visão que melhor expresse a continuidade de valores no banco Pima Indians, foram feitos vários ensaios estatísticos.

Nesse estudo, para apresentar uma melhor demonstração das formas de extração dos Graus de Evidência, utilizou-se apenas a frequência de Glicemia; no entanto, verifica-se que os mesmos processos de análise poderão ser facilmente expandidos para os outros fatores extraídos do Banco de Dados. 
Entre os resultados estatísticos mais significativos, estabeleceram-se critérios para a escolha do gráfico e a sua correspondente tabela $2 \times 2$, que seja mais adequada aos parâmetros estabelecidos nos diagnósticos médicos a serem utilizados como exemplo para esse estudo, quando ocorre uma divisão quase por igual de doentes e não doentes em PC $=110 \mathrm{mg} / \mathrm{dl}$.

$\mathrm{Na}$ análise da Glicemia para 100 mg/dl foi encontrada uma Prevalência de 74,3490\%, e para $110 \mathrm{mg} / \mathrm{dl}$, encontrou-se 59,115\%, que se refere a uma redução considerável. Verificou-se que para $140 \mathrm{mg} / \mathrm{dl}$ a Prevalência reduz ainda mais, a porcentagem vai para $25,651 \%$.

Dessa forma, na análise inicial dos resultados estatísticos, verifica-se que $110 \mathrm{mg} / \mathrm{dl}$ (Figura 2) é o valor que melhor representa essa situação, segundo visto nos Histogramas citados. (SOUZA, 2009)

Seguindo os critérios e os resultados das análises iniciais, optou-se pela utilização do gráfico (e tabela 2x2) com Ponto de Corte $110 \mathrm{mg} / \mathrm{dl}$, cujo resultado está exposto na figura 2 a seguir.

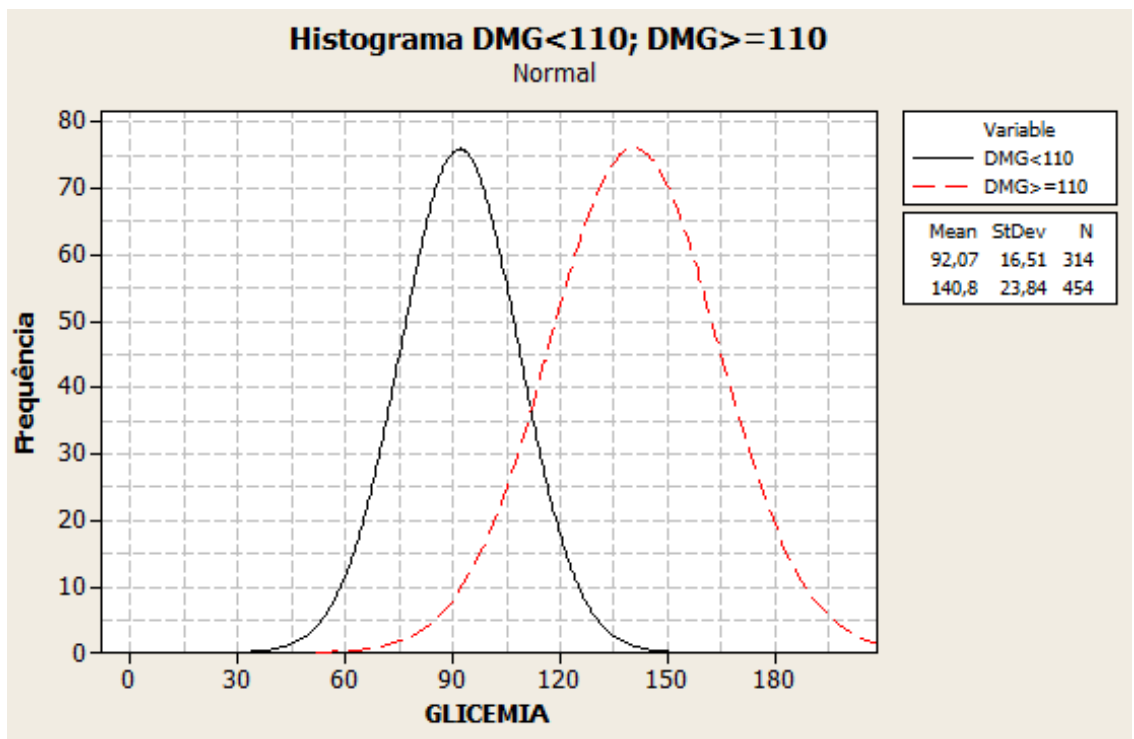

Figura 2 - Gráficos das Normais para Glicemia 110 mg/dl [15]

Com a definição do Gráfico, em seguida foi determinado o intervalo de interesse, ou o Universo de Discurso ${ }^{2}$ para a extração dos Graus de Evidência. Para o gráfico que representa as características no ponto de corte $110 \mathrm{mg} / \mathrm{dl}$ o UD foi escolhido pela variação máxima entre os picos das formas de onda.

O Intervalo que define o Universo de Discurso do estudo é mostrado pelas linhas tracejadas no gráfico da figura 3. 


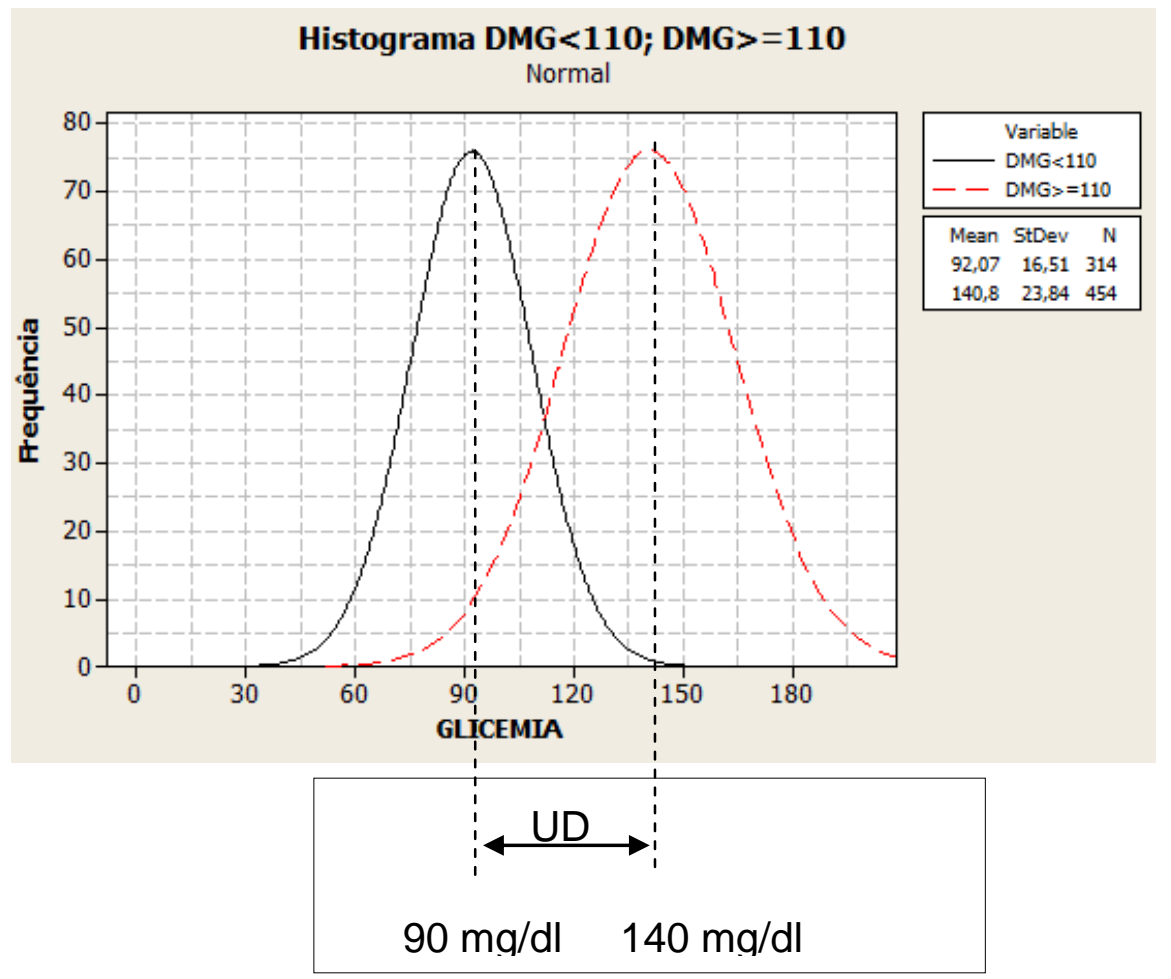

Figura 3 - Gráficos das Normais para Glicemia, 90/140 mg/dl [15]

Dessa forma, verifica-se que o intervalo de interesse no gráfico Frequência x Glicemia vai de um valor em torno de 90 até $140 \mathrm{mg} / \mathrm{dl}$ de Glicemia. Esses dados para o Ponto de Corte $110 \mathrm{mg} / \mathrm{dl}$ serão considerados para a obtenção dos Graus de Evidência, conforme descrito a seguir.

\section{MÉTOdO DE EXTRAÇÃO DO GRAU DE EVIDÊNCIA A PARTIR DAS FREQUÊNCIAS E DESVIOS}

Nesse modo, a partir dos valores estatísticos de frequência serão extraídos os dados que, após receberem um tratamento lógico paraconsistente, resultarão em Graus de Evidência $\mu_{\mathrm{EF}}$. Para a demonstração desse método utilizaremos o gráfico que mostra as variações de frequência e Glicemia no banco de dados Índias Pima. [14]

O método de determinação de Grau Evidência utilizando valores estatísticos se refere ao cálculo de variáveis de distribuição Normal com aplicações da LPA. Essa metodologia se destina a determinar o Grau de Evidência Favorável em DMG pela Glicemia.

Para a extração do Grau de Evidência, iremos a partir do resultado do gráfico obtido fazer a caracterização das curvas com simetria em torno da Média.

Para abranger um intervalo que compreende praticamente $100 \%$ de probabilidade sob a curva [2] vamos considerar, como análise além da Média, o triplo do Desvio Padrão. 
Podemos acomp

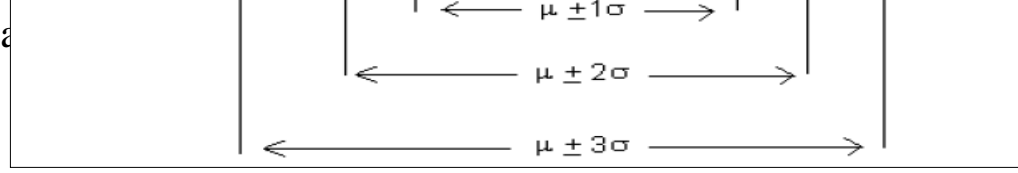

Figura 4. Definição de valores de Desvio Padrão da

Curva Normal ou de Gauss [3]

Sendo assim estaremos considerando [16]:

[ $\mu$ - 3 x desvios-padrões; $\mu+3 \times$ desvios-padrões]

Ou seja

[ ${ }^{X}-3$ x desvios-padrões $(\sigma) ;{ }^{X}+3$ x desvios-padrões $\left.(\sigma)\right]$

Para a curva normal, temos estatisticamente a seguinte formulação da função:

$$
\begin{aligned}
& \boldsymbol{f}(\mathbf{x})=\frac{1}{\sigma \sqrt{2 \Pi}} e^{\frac{-(x-\mu)^{2}}{2 \sigma^{2}}}, \mathbf{x} \in R \\
& (-\infty<\boldsymbol{\mu}<+\infty ; \boldsymbol{\sigma}>\mathbf{0})
\end{aligned}
$$

Fonte das fórmulas: [3]

\subsection{TÉCNICA DE EXTRAÇÃO DOS GRAUS DE EVIDÊNCIA}

Segundo Da Silva Filho et al. [7], sistemas de apoio à tomada de decisão que tratam com o Conhecimento Incerto devem ser capazes de representar, manipular e comunicar dados considerados imperfeitos.

Sabemos que na estatística trabalhamos com dados incertos e portanto muitas vezes imprecisos; em diagnóstico médico podemos ter as seguintes considerações:

Um sistema de apoio à tomada de decisão deve ser robusto o suficiente e bem fundamentado para responder a critérios teóricos, portanto necessita estar subsidiado por uma teoria adequada de incerteza que viabilize, dentro de determinados limites, qualquer verificação, independentemente do domínio da aplicação. [7]

O sistema deve ser capaz de apresentar boa computabilidade dos dados envolvidos. No tratamento de incertezas, os valores devem ser computacionalmente factíveis para o sistema criar regras de inferências e obter conclusões. No tratamento de dados com valores incertos, o sistema deve viabilizar a combinação de avaliações qualitativas com valores quantitativos de incerteza.

Veremos agora uma técnica de modelagem de sinais (dados) que irão representar as evidências sobre as grandezas a serem analisadas pela rede. A partir desses dados vamos usar 
uma modelagem que seja compatível com os gráficos das Normais, Gráfico da Figura 4, escolhido o Histograma de valor $110 \mathrm{mg} / \mathrm{dl}$ por similaridade de representação. [14]

Conforme verificamos para um Ponto de Corte de $110 \mathrm{mg} / \mathrm{dl}$ o banco Índias Pima gerou o Gráfico abaixo na Figura 5, onde podemos verificar a frequência dos picos, bem como seus desvios.

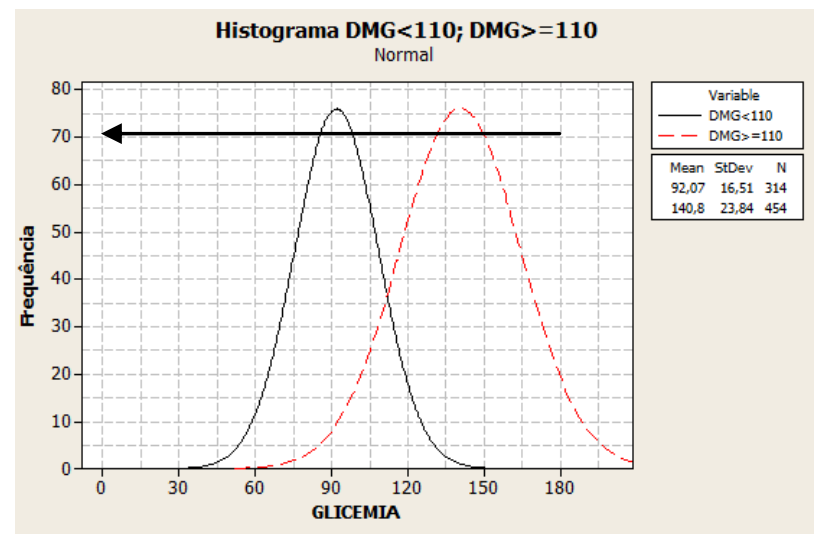

Figura 5. Glicemia em $110 \mathrm{mg} / \mathrm{dl}$ (análise em andamento). [15]

Consideremos inicialmente a mesma proposição para a extração do Grau de Evidência: $\mathrm{P}$ “O Paciente está com diabetes"

Nesse gráfico podemos considerar a variação dos Graus de Evidência Favorável dado a variação da frequência na proposição analisada, conforme indica a figura a seguir:

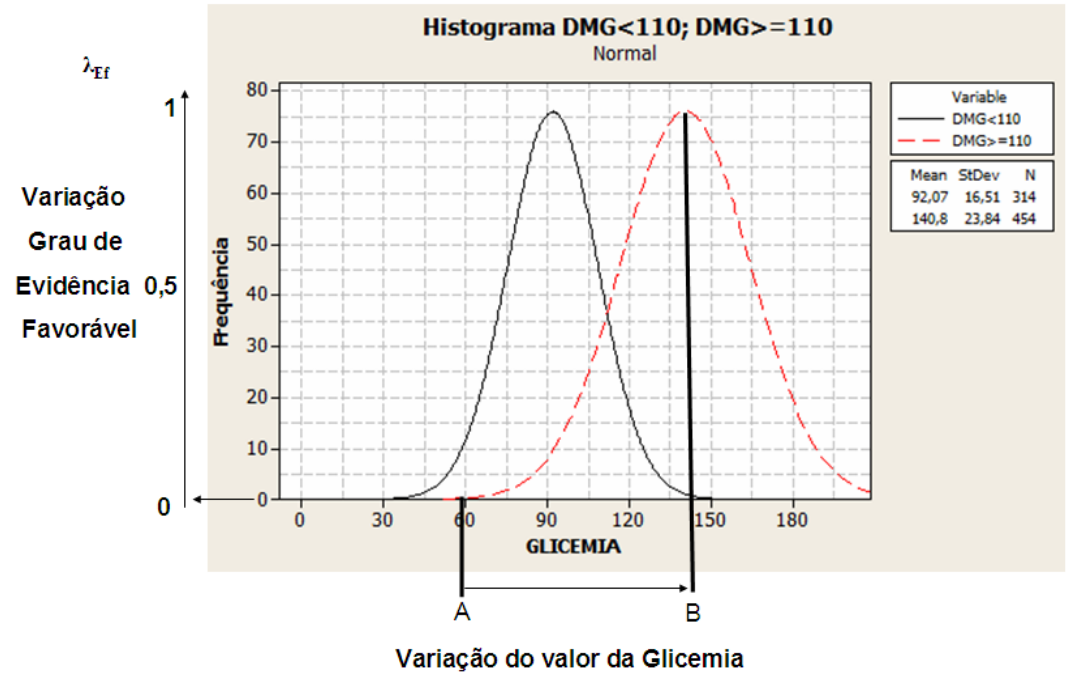

Figura 6. Glicemia em 110 mg/dl (Análise A-B) Positivo. [15]

Da mesma forma podemos considerar a variação dos Graus de Evidência Desfavorável à proposição analisada, conforme indica a figura a seguir: 


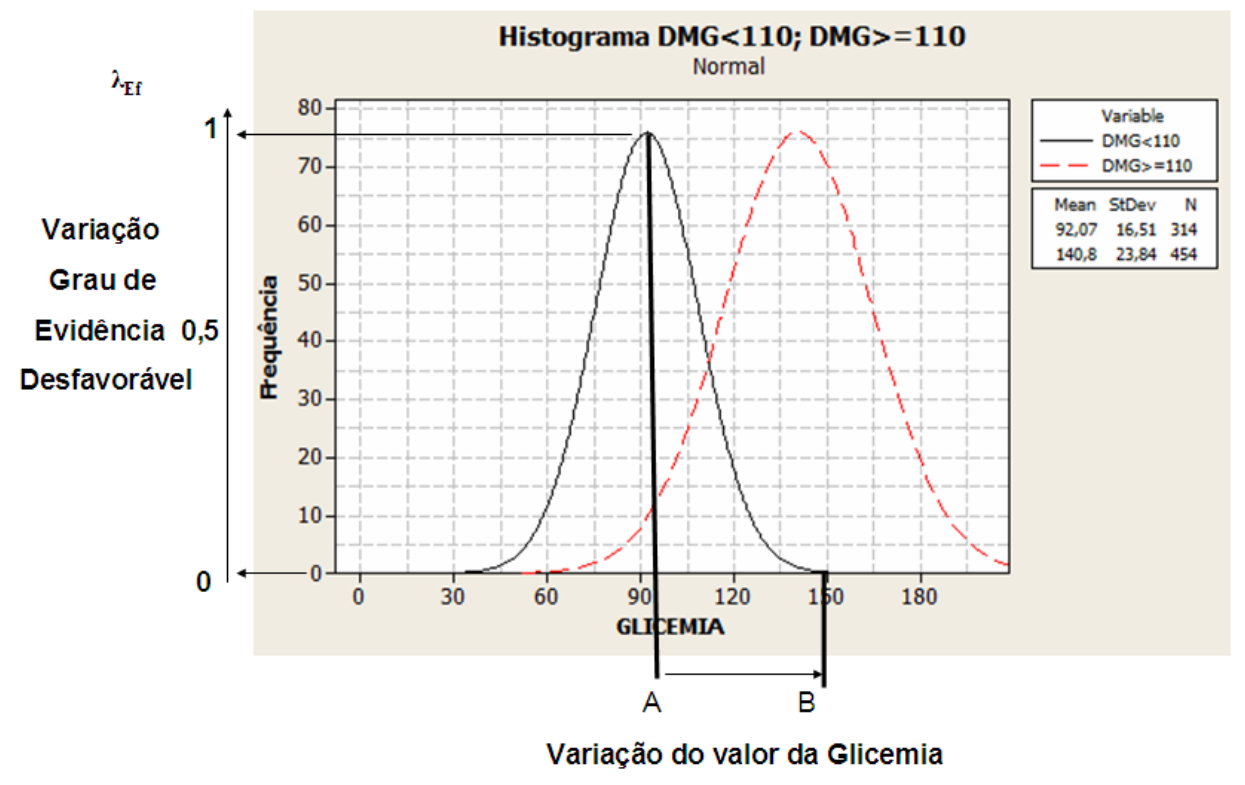

Figura 7. Glicemia em 110 mg/dl (análise A-B) Negativo. [15]

Para na modelagem encontrar os valores dos Graus de Evidência intermediários, foi escolhida uma função ou semelhante, adequada à variação do banco de dados Pima Indians . A função escolhida foi a de variação do tipo S, mostrada no Gráfico da figura 8. A adequação foi feita do seguinte modo:

Considere um Universo de Discurso que vá do valor-limite inferior da medição de grandeza, simbolizado por $\mathrm{a}_{1}$, até o valor do limite superior da medição da grandeza, simbolizado por $\mathrm{a}_{2}$, com uma variação não-linear das medições da grandeza na forma de função S. [7].

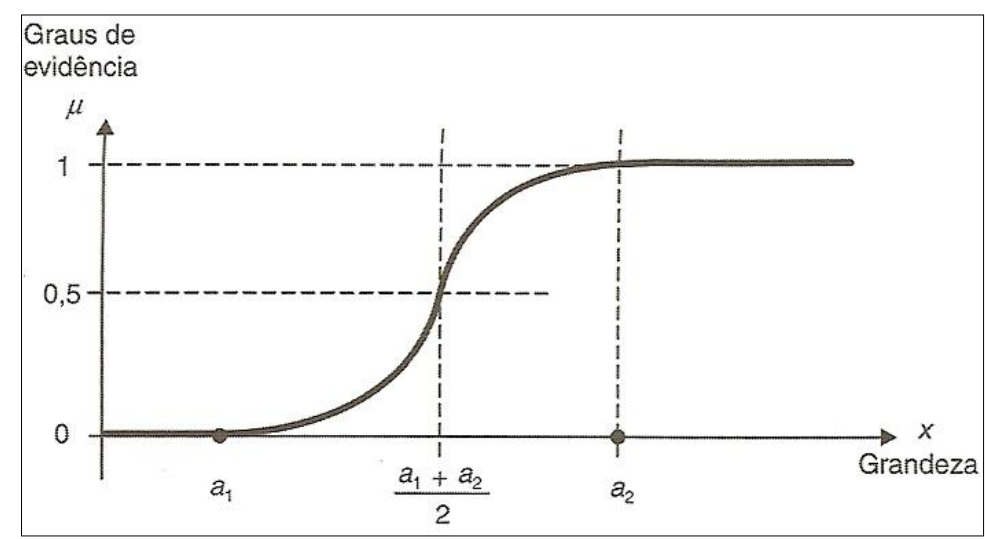

Figura 8. Validação do Grau de Evidência com variação conforme a função S. [7]

Para $\mu(\mathrm{X})$ teremos a seguinte tabela abaixo: 
Tabela 2. Formulário para o lado Positivo. [7]

\begin{tabular}{|c|c|c|c|}
\hline \multirow{2}{*}{$\boldsymbol{\mu}_{(\mathbf{x})}$} & $\mathbf{0}$ & $\mathrm{Se}$ & $\mathbf{x}<a_{1}$ \\
\cline { 2 - 4 } & $\mathbf{2}\left(\frac{x-a_{1}}{a_{2}-a_{1}}\right)^{2}$ & Se & $a_{1} \leq \mathbf{x} \leq\left[\frac{a_{1}+a_{2}}{2}\right]$ \\
\cline { 2 - 4 } & $1-2\left(\frac{x-a_{2}}{a_{2}-a_{1}}\right)^{2}$ & Se & {$\left[\frac{a_{1}+a_{2}}{2}\right]<\mathbf{x} \leq a_{2}$} \\
\hline 1 & Se & $\mathbf{x}>a_{2}$ \\
\hline
\end{tabular}

Da mesma forma que foi padronizada a função S, Gráfico da Figura 9, pode-se obter outra função diferente, que chamaremos de função $Z$, que é o complemento da função anterior.

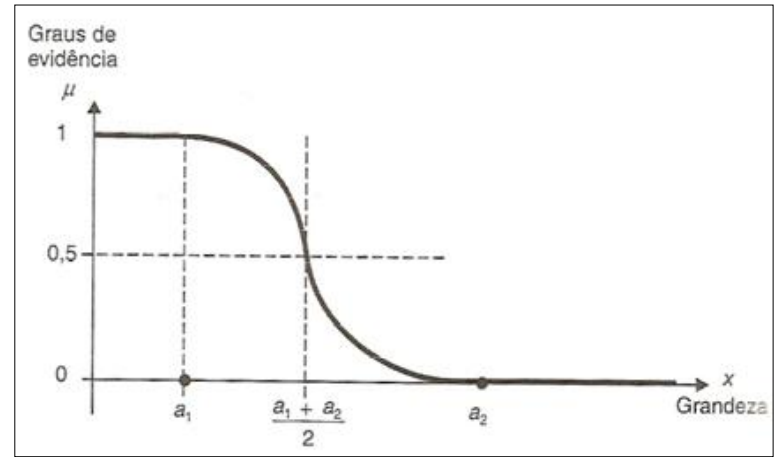

Figura 9. Valoração do grau de Evidência com variação conforme a função Z. [7]

Podemos descrever suas equações da seguinte forma:

Tabela 3. Formulário para o lado Negativo. [7]

\begin{tabular}{|c|c|c|c|}
\hline \multirow{2}{*}{$\boldsymbol{\mu}_{(\mathbf{x})}$} & $\mathbf{0}$ & Se & $\mathbf{x}>a_{2}$ \\
\cline { 2 - 4 } & $1-2\left(\frac{x-a_{1}}{a_{2}-a_{1}}\right)^{2}$ & Se & $a_{1} \leq \mathbf{x} \leq\left[\frac{a_{1}+a_{2}}{2}\right]$ \\
\cline { 2 - 4 } & $\mathbf{2}\left(\frac{x-a_{2}}{a_{2}-a_{1}}\right)^{2}$ & Se & {$\left[\frac{a_{1}+a_{2}}{2}\right] \leq \mathbf{x} \leq a_{2}$} \\
\hline 1 & Se & $\mathbf{x}<a_{1}$ \\
\hline
\end{tabular}

A extração do Grau de Evidência favorável $\mu_{\mathrm{Ef}}$ e o Grau de Evidência Desfavorável $\lambda_{\text {Ef }}$ permite que estes dois valores que estão no do intervalo [0,1] sejam analisados por um Nó de Análise Paraconsistente (NAP). Desse modo qualquer valor de Glicemia será transformado 
em um Grau de Evidência Favorável e um Grau de Evidência Desfavorável e, através do NAP, será obtido o Grau de Evidência Real. A figura 10 a seguir mostra essa análise:

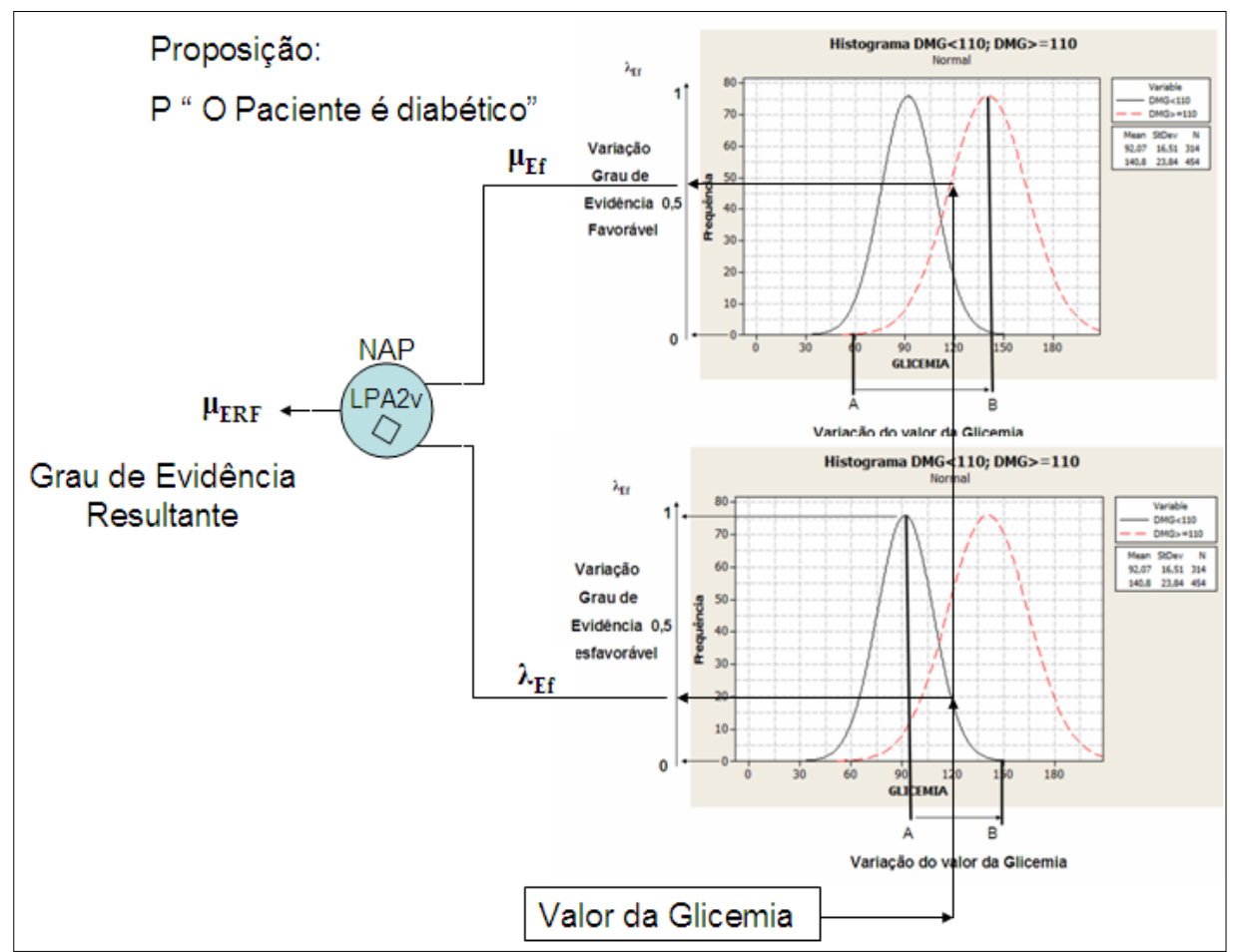

Figura 10. Análise do Grau de Evidência Resultante. [15]

Um programa em linguagem $C$ foi desenvolvido pelo autor para fazer esse tipo de extração de Grau de Evidência para apoio ao diagnóstico médico (APÊNDICE B).

A princípio, foram analisados os valores pré-definidos de Glicemia, para a construção do gráfico de Normais, conforme intervalo do banco de dados das Índias Pima, com PC 110 $\mathrm{mg} / \mathrm{dl}$.

Verifica-se que para essa metodologia de aplicação de cálculo de Evidência estaremos selecionando as áreas conforme mostrado nos Gráficos das Figuras 11 e 15 (Negativo e Positivo), a seguir.

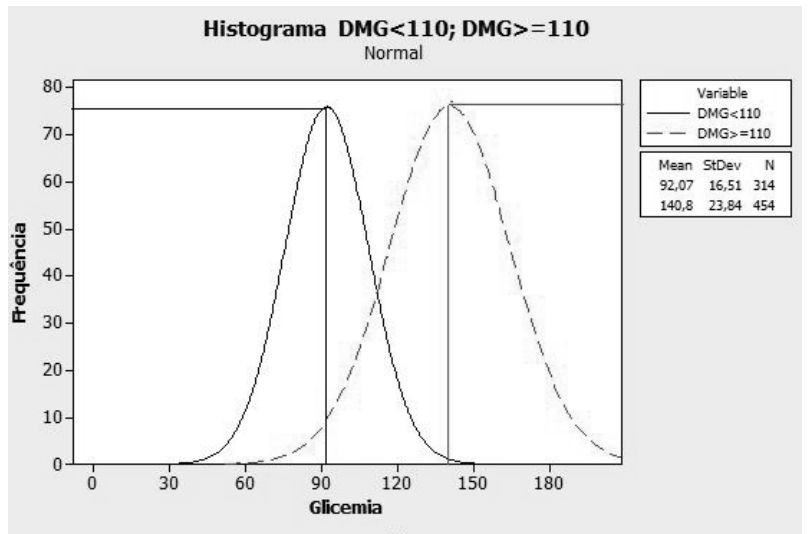

Figura 11. Delineando as regiões e os picos para valor $=1$. [15] 


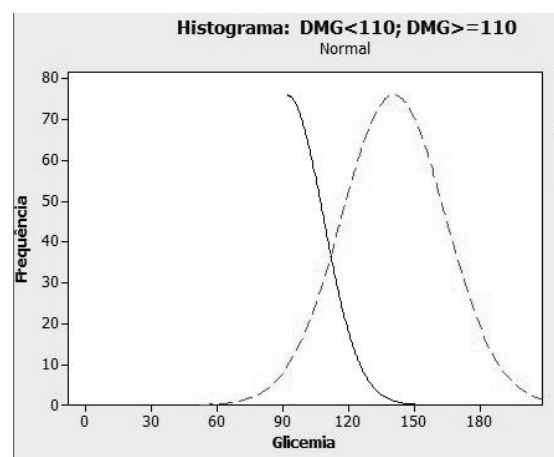

Figura 12. Área suprimida não utilizada da curva Negativa.[15]
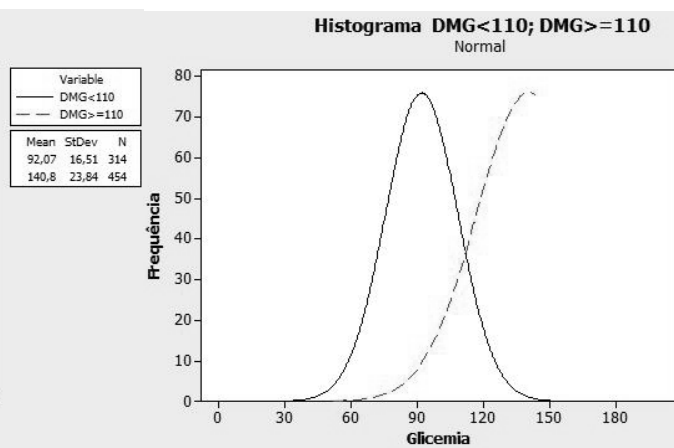

Figura 13. Área suprimida não utilizada da curva Positiva.[15]

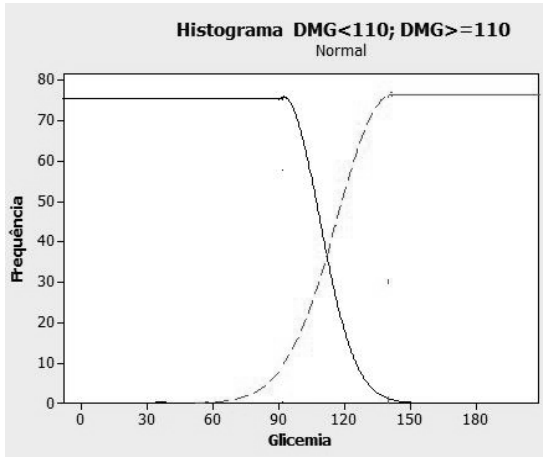

Figura 14. Regiões delineadas como Negativa e Positiva.[15]

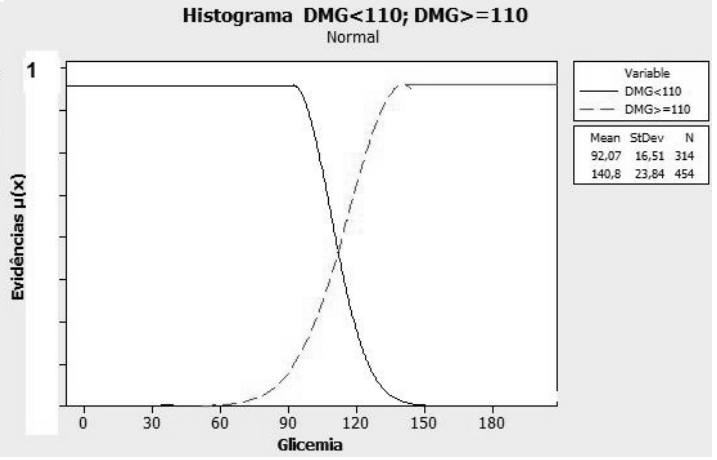

Figura 15. Evidência vs Glicemia. [15]

Tabela 3. Cálculo da Evidência

\begin{tabular}{ccccccccc}
\hline EXP_N & STATUS & GLICEMIA & $\mathbf{X}$ & $\mathbf{S}$ & MULHERES & $\boldsymbol{\mu}$ & EVIDÊNCIA FAVORÁVEL \\
\hline $\mathbf{1}$ & NEGATIVO & 90 & 75,84 & 19,09 & 104 & 0,878 & NEGATIVO & $\mu \mathrm{a}$ \\
\hline & POSITIVO & & 128 & 27,53 & 664 & 0,577 & & $\mu \mathrm{b}$ \\
\hline $\mathbf{2}$ & NEGATIVO & 95 & 79,05 & 18,52 & 504 & 0,835 & NEGATIVO & $\mu \mathrm{a}$ \\
\hline & & & & & & & & \\
\hline & POSITIVO & & 130,5 & 26,71 & 621 & 0,607 & & $\mu \mathrm{b}$ \\
\hline $\mathbf{3}$ & NEGATIVO & 100 & 84,7 & 16,83 & 197 & 0,816 & NEGATIVO & $\mu \mathrm{a}$ \\
\hline & & & & & & & $\mu \mathrm{b}$ \\
\hline & POSITIVO & & 133,4 & 25,87 & 571 & 0,630 & & $\mu \mathrm{a}$ \\
\hline
\end{tabular}




\begin{tabular}{|c|c|c|c|c|c|c|c|c|}
\hline & POSITIVO & & 136,7 & 24,94 & 517 & 0,641 & & $\mu b$ \\
\hline \multirow[t]{2}{*}{5} & NEGATIVO & 110 & 92,07 & 16,51 & 314 & 0,738 & NEGATIVO & $\mu \mathrm{a}$ \\
\hline & POSITIVO & & 140,8 & 23,84 & 454 & 0,629 & & $\mu \mathrm{b}$ \\
\hline \multirow[t]{2}{*}{6} & NEGATIVO & 115 & 94,76 & 16,81 & 363 & 0,678 & NEGATIVO & $\mu \mathrm{a}$ \\
\hline & POSITIVO & & 144,3 & 22,89 & 405 & 0,636 & & $\mu \mathrm{b}$ \\
\hline \multirow[t]{2}{*}{7} & NEGATIVO & 120 & 97,22 & 17,33 & 406 & 0,616 & & $\mu \mathrm{a}$ \\
\hline & POSITIVO & & 147,7 & 22,01 & 360 & 0.648 & POSITIVO & $\mu b$ \\
\hline
\end{tabular}

Legenda - Tabela:

Exp_N: Número De Amostras

Status: Resultado: Negativo Ou Positivo tabela

Glicemia para DMG: Dados de Corte Em mg/dl

X: Valor Médio; S: Desvio Padrão; Mulheres: Quantidade

$\mu_{\mathrm{a}}$ : Evidência Da Fonte A; $\mu_{\mathbf{b}}$ : Evidência Da Fonte B

$\boldsymbol{\mu}$ : Evidência Favorável; $\boldsymbol{\lambda}$ : Evidência Desfavorável

Pelos valores obtidos para análise de Glicemia $=90 \mathrm{mg} / \mathrm{dl}$

Foi calculado:

$\mu_{\mathrm{a}}=0,878$ e $\mu_{\mathrm{b}}=0,577 \Rightarrow \mu_{\mathrm{a}}<\mu_{\mathrm{b}}$

Verificamos que enquanto o Grau de Evidência Favorável é Negativo, não prevalece a diabetes; quando se inverte para positivo, teremos o fator favorável à doença. [14]

\section{CONCLUSÃO}

Neste artigo procuramos apresentar métodos para analisar conceitos teóricos da Lógica e construir o elo com o trabalho científico de investigação, objetivando a aplicação da Lógica Paraconsistente Anotada com dois valores (LPA2v) em aplicações inovadoras na área da Saúde, como a Probabilidade Pragmática, técnicas de Modelagens dos Sinais Lógicos Paraconsistentes e Aplicações de Valores de Prevalência Positiva. Essas técnicas inseridas em 
ferramentas computacionais agregam valores formativos altamente positivos e seguros quanto à tomada de decisão.

A explosão de novos e dispendiosos testes diagnósticos exige dos profissionais de saúde que, além do bom senso, aprendam e utilizem de forma eficiente a estratégia dos fatores conhecidos como prevalência, sensibilidade, especificidade, valores preditivos e razões de probabilidades, pois são instrumentos úteis na avaliação das dezenas de testes diagnósticos que aparecem diariamente nas revistas e consultórios médicos.

Ao clínico compete decidir se vale a pena usá-los ou não. Nessa análise, os benefícios clínicos devem ser cortejados com os riscos médicos, o ônus econômico e as vantagens e desvantagens em relação aos outros exames. Embora não seja uma tarefa fácil, é dever do médico conhecer as técnicas de validação e interpretação dos testes diagnósticos, para decidir em bases científicas sua real utilidade.

\section{REFERÊNCIAS}

[1] ABE, J. M. Fundamentos da Lógica Anotada.1992. Tese de Doutorado, Universidade de São Paulo, FFLCH/USP - São Paulo, 1992.

[2] BEIGUELMAN, B. Curso prático de Bioestatística. 3.a Ed. Ver., Ribeirão Preto, Editora Revista Brasileira de Genética, 1994.

[3] BITTENCOURT, H. R. Estatística Aplicada a Psicologia. Pontifícia Universidade Católica do Rio Grande do Sul. Faculdade de Matemática. Departamento de Estatística. Apostila online em pdf, 2006.

[4] DA SILVA FILHO, J. I. Métodos de Aplicações da Lógica Paraconsistente Anotada de anotação com dois valores LPA2v com construção de Algoritmo e Implementação de Circuitos Eletrônicos. 1999. Tese de Doutorado. EPUSP, São Paulo, 1999.

[5] DA SILVA FILHO, J. I. \& ABE, J. M. “Introdução à Lógica Paraconsistente Anotada” Editora Emmy - 1. ${ }^{\text {a }}$ Edição, 2000.

[6] DA SILVA FILHO, J. I. \& ABE, J. M. "Fundamentos das Redes Neurais Paraconsistentes". Destacando Aplicações em Neurocomputação. Editora Arte \& Ciência, ISBN 85-7473-045-9, 247 pp., 2001.

[7] DA SILVA FILHO, J. I. "Lógica Paraconsistente e Probabilidade Pragmática no Tratamento de Incertezas". Revista Seleção Documental n. 9, - p 16-27 ano 3 - ISSN 18090648, Jan/Fev/Mar, 2008.

[8] DA SILVA FILHO, J.I. et al. ;ABE, J.M.; TORRES, G. L. "Inteligência Artificial com Redes de Análises Paraconsistentes. Teorias e Aplicações”. Ed.LTC. R.J., 2008.

[9] DIABETES NEWS. REVISTA. SOCIEDADE BRASILEIRA DE DIABETES $(S B D)$. VOLUME 1. NÚMERO 2. EDITORA: DIGRAPHIC. Ago, 2004.

[10] HECHT-NIELSEN, R. “Neurocomputing”. New York, Addison Wesley Pub. Co.,1990. 
[11] MALINOWSKI, BK.Argonautas do Pacífico Ocidental. Um relato do empreendorismo e da aventura dos nativos nos arquipélagos da Nova Guiné Melanésia. 3.a edição. Abril Cultural. São Paulo, 1984

[12] MARQUES, R.L.; DUTRA,I. “Redes Bayesianas: o que são, para que servem, algoritmos e exemplos de aplicações". COPPE Sistemas - UFRJ

[13] SigILliTO, V. "Pima Indians Diabetes Database", fornecida pela John Hopkins University, 1990. Propriedade do National Institute of Diabetes and Digestive and Kidney Diseases. Doado por Vincent Sigillito (vgs@aplcen.apl.jhu.edu), Research Center, RMI Group Leader, Applied Physics Laboratory, The Johns Hopkins University - em maio/1990. Disponível em:

<http://www.teco.uni-karlsruhe.de/ albrecht/neuro/src/testdata/diab/diab.txt>. .

Acesso em: 13. Jan. 2005.

[14] SOUZA, P.R.S. "MÉTODOS DE APOIO A DECISÃO MÉDICA PARA ANÁLISE EM DIABETES MELLITUS GESTACIONAL UTILIZANDO A PROBABILIDADE PRAGMÁTICA NA LÓGICA PARACONSISTENTE ANOTADA DE DOIS VALORES PARA MELHOR PRECISÃO DE RESPOSTA”, TESE DE DOUTORADO. POLITÉCNICA USP, ABRIL DE 2009.

[15] MINITAB INC. LEAD TECHNOLOGIES, INC. SOFTWARE. VERSÃO 15.1.30.0, 2007.

[16] MASSAD, E.; DE MENEZES, R. X.;.SILVEIRA, P. S. P.; ORTEGA, N. R. S. ’Métodos Quantitativos em Medicina”, Editora Manole Ltda. - 1. ' Edição, 2004.

\section{APÊNDICE A - ALGORITMO DE BAYES ALGORÍTMO QUE FORNECE O RESULTADO DO CÁlCULO DO VALOR DA PREVALÊNCIA, SENSIBILIDADE, ESPECIFICIDADE E FALSO-POSITIVO (1-ESPECIFICIDADE). ARQUIVO: BAYES.C}

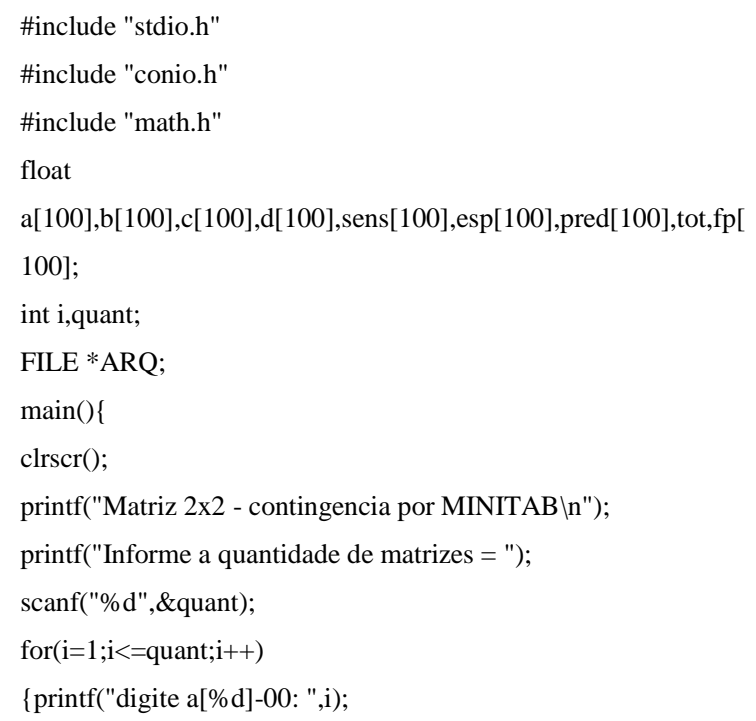

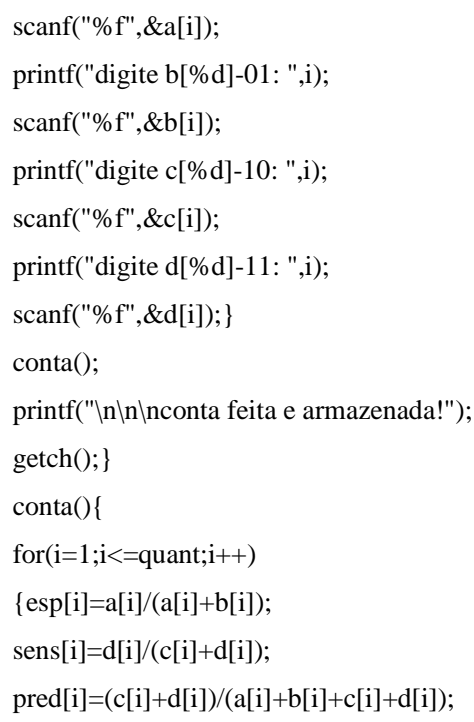


fp[i]=1-esp[i];\}

ARQ=fopen("bayes.txt","w");

for $(\mathrm{i}=1 ; \mathrm{i}<=$ quant;i+++) $\{$ fprintf(ARQ,"\%f\%f\%f\%fln",sens[i],esp[i

],

$\operatorname{pred}[\mathrm{i}], \mathrm{fp}[\mathrm{i}])$; $\operatorname{printf}(" \ln \backslash n s e n s=\%$ f, $\quad$ esp=\%f, $\quad$ pred=\%f,

$\mathrm{fp}=\% \mathrm{f}^{\prime}$,sens[i],esp[i],pred[i],fp[i]);

\}fclose(ARQ);

\}

Algoritmo, Adaptação e Codificação: do Autor, 2008.

\section{APÊNDICE B - ALGORITMO DO NAP USANDO GLICEMIA, VALOR MÉDIO E DESVIO PADRÃO}

Algoritmo NAP na Linguagem C que fornece o cálculo do valor do Grau Evidência Favorável e Desfavorável como resultado da Análise (positivo e negativo).

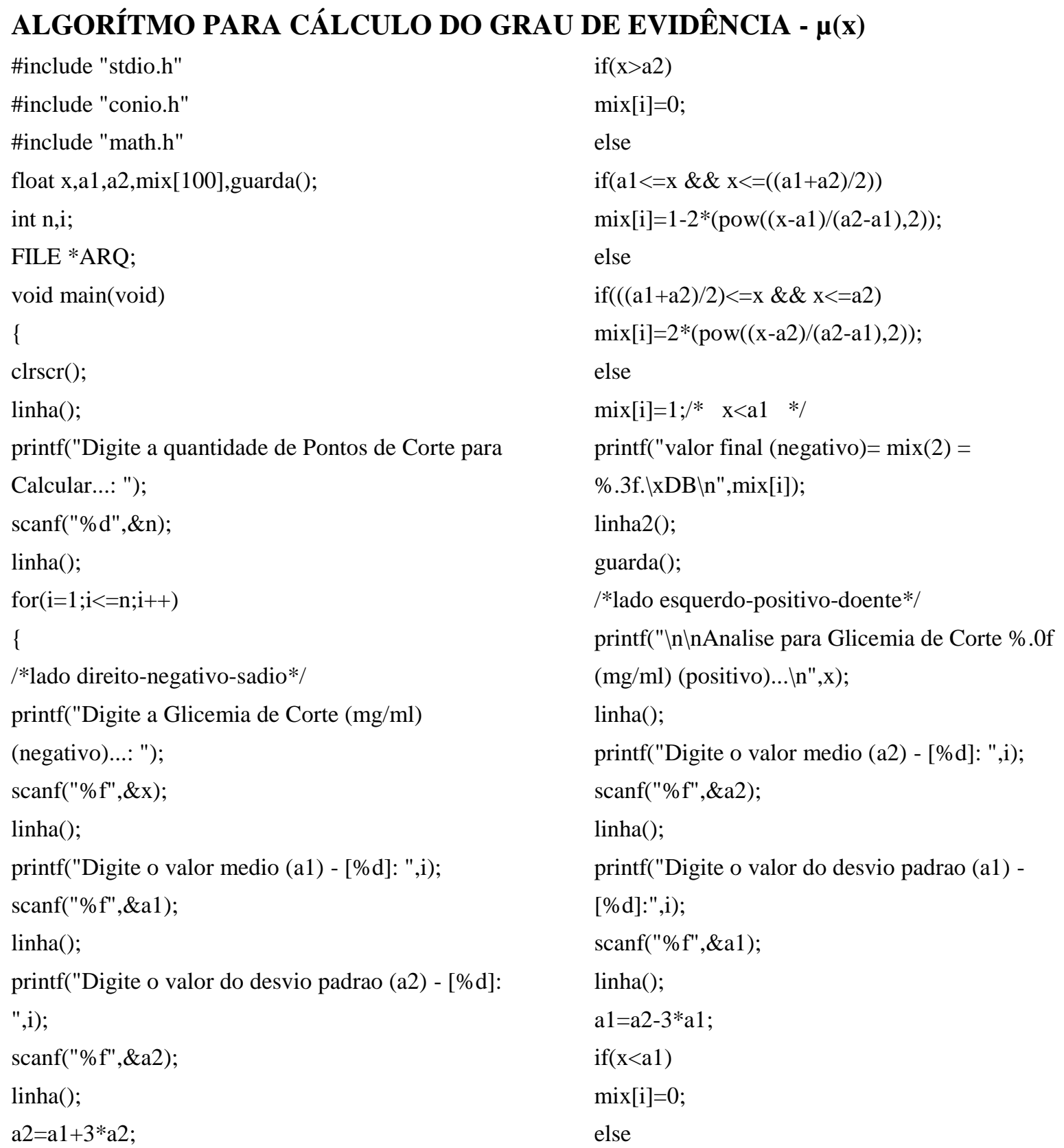




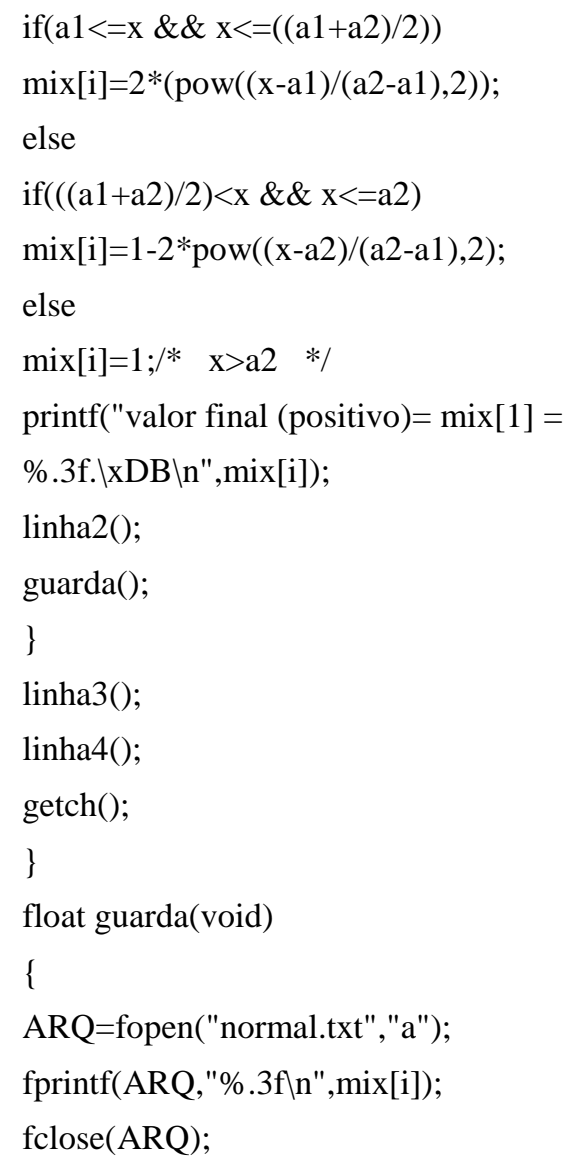

Formulário: Fonte: (Da Silva Filho et. al., 2008, p.89-90)

Adaptação, Algoritmo e Codificação: do Autor, 2008. 